Neurosurg Focus 13 (6):Article 2, 2002, Click here to return to Table of Contents

\title{
Bone morphogenetic proteins: basic concepts
}

\author{
SETTI S. Rengachary, M.D. \\ Department of Neurosurgery, Wayne State University School of Medicine, Detroit Medical Center, \\ Detroit, Michigan
}

\begin{abstract}
The cellular and molecular events governing bone formation in the embryo, healing of a fractured bone, and induced bone fusion follow a similar pattern. Discovery, purification, and recombinant synthesis of bone morphogenetic proteins (BMPs) constiute a major milestone in the understanding of bone physiology. In this review the author discusses the mechanism of action, clinical applications, dosage, and optimum carriers for BMPs. The roles played by other growth factors are also discussed.
\end{abstract}

\section{KEY WORDS • bone healing • bone morphogenetic protein • carrier • growth factors - spinal fusion}

Bone is unique of all the tissues in the in the vertebrate organism. When injured, it heals by formation of new bone. In contrast, most other tissues such as the heart muscle, voluntary muscles, liver, and the brain heal by replacement of connective tissue rather than the original tissue.

Another interesting attribute of the bone is that the molecular and cellular processes that lead to the development of the skeletal structures within the embryo are very similar to the cascades that occur in the healing process in an injured bone. Likewise, in surgically created fusion, the osseous fusion mass formation recapitulates a fracture healing process, which in turn recapitulates embryonic development of new bone. Thus, there is a common theme in the development of bone from primitive mesenchymal tissues to a well-structured, well-organized histological structure that one associates with mature bone. In addition, the ongoing remodeling process in an adult organism, which is exposed to external physical and hormonal influences, is also modulated through a similar molecular mechanism.

The developing limb bud represents a prototypical example of bone development in the embryo. There is a condensation of primitive mesenchymal cells in the central core of the limb bud, in the area destined to form skeletal

Abbreviations used in this paper: $\mathrm{BMP}=$ bone morphogenetic protein; $\mathrm{ECM}=$ extracellular matrix; FDA = Food and Drug Administration; $\mathrm{OP}=$ osteogenic protein $; \mathrm{PDGF}=$ platelet-derived growth factor; $\mathrm{TCP}=$ tricalcium phosphate; $\mathrm{TGF}=$ transforming growth factor. structures. This condensation usually transforms into bone through two independent pathways. Intramembranous ossification occurs when there is a direct ossification of the mesenchymal tissues. The primitive mesenchymal cells are transformed into osteoprogenitor cells and then into mature osteoblasts leading to the formation of the bone with all of its histological characteristics. ${ }^{11}$ This process occurs typically in the calvarial bones, mandible, and the clavicle. In contrast, the epiphysial growth plate in the appendicular skeleton is characterized by the intracartilaginous bone formation. In this process the primitive mesenchymal cells differentiate in a two-step process into mature bone. In the first step, the mesenchymal cells transform into chondroblasts, form collagen and other elements of bone matrix, become ossified, and lead to mature bone. In the embryonic phase a formation of bone through intramembranous compared with intracartilaginous process depends on the anatomical site. As stated earlier, the cranial structures and mandible undergo an intramembranous growth process whereas the appendicular skeleton forms through an intracartilaginous process.

The postfracture healing of bone generally follows in the intracartilaginous ossification process, although with a very high concentration of BMP an intramembranous route may be taken. ${ }^{20}$ At this time, it is unclear what factor(s) direct(s) one process (intramembranous, for example) as opposed to the other in the embryonic phase or during fracture healing. Because the bone that is formed as a result of either process is virtually indistinguishable in its final mature form, it is unclear what the biological advantage is of one process over the other. 


\section{THE CASCADE OF BONE HEALING FOLLOWING FRACTURE}

The stages of bone healing/remodeling are summarized in Table 1. Immediately following a fracture, a hematoma forms at the fracture site due to injury to the periosteum and the adjacent soft tissues. The repair process is initiated with the macrophages are attracted by chemotaxis to the injured site. ${ }^{3}$ Macrophages remove the tissue debri whereas fibroblasts generate ECM. ${ }^{13}$ A decrease in local oxygen tension and loss of nutrients are factors that promote the release of cytokines and growth factors at the fracture site (Table 2). Degranulating platelets in the hematoma release PDGF and TGF 3 . Interleukin- 1 and -6 are also released by the inflammatory cells. ${ }^{2,35}$ Because of the mitogenic activity of the TGF $\beta$ and PDGF, mesenchymal cells and fibroblasts proliferate at the fracture site. ${ }^{29}$ The sources of the mesenchymal stem cells are the bone marrow, periosteum, and surrounding soft tissues. Many of these mesenchymal cells are transformed into osteoprogenitor cells by the locally expressed BMPs of various types. ${ }^{36}$ Initially chondrogenesis occurs resulting in a soft callus. Calcification of the ECM ensues. ${ }^{15-19}$ Angiogenesis is induced concurrently and leads to the formation of woven bone, which then matures into lamellar architecture of the fully developed bone and bone marrow. Remodeling of the bone completes the process.

\section{HISTORY OF BONE MORPHOGENETIC PROTEIN}

The history of BMPs is highlighted in Table 3. As early as 1889 , Senn ${ }^{27}$ noticed that decalcified bone can induce healing of bone defects. He was treating osteomyelitic defects in the bone by using decalcified residue of ox bone with iodoform. His primary goal was use of iodoform as an antiseptic to treat the osteomyelitis, and the decalcified ox bone as was intended a carrier for the iodoform. Serendipitously he noticed that not only was the infection controlled but new bone formed at the osseous defect. Although the observation was interesting, his findings were not easily reproduced by others.

In the 1930 s, Levander ${ }^{9,10}$ noted that crude alcohol extracts of bone induced new bone formation when injected into muscle tissue. In 1961 Sharrard and Collins ${ }^{28}$ reported the use of ethylenediaminetetraacetic acid-decalcified allograft bone for spinal fusion in children. This idea was supported by contemporaneous laboratory studies by Ray and Holloway. ${ }^{14}$

The seminal discovery of the ability of the bone matrix to induce bone was made by Urist in $1965 .{ }^{30}$ Urist was director of the bone research laboratory at the University of California, Los Angeles School of Medicine, and was a practicing orthopedic surgeon. He showed that crude bone extracts induced new bone in an ectopic site (in a muscle pouch) in a rat model. He coined the term "bone morphogenetic protein" or "osteogenic protein" which was the active ingredient contained in this extract. His research, however, was hampered by the fact that there was no reproducible assay for the protein. Additionally, it was not conclusively determined that this putative protein was responsible for the induction of new bone in an ectopic site. That task was accomplished by Reddi and Sampath in 1983 when they invented a crude but highly reproducible assay for ectopic bone formation. The assay was based on the activity of alkaline phosphatase and the Ca content of the newly formed bone. This group also showed that when the protein component was dissociated from the matrix, ${ }^{23}$ the remaining matrix in itself did not induce new bone formation. When the matrix was reconstituted with the protein, however, it was quite effective as the original matrix in inducing the bone. This conclusively proved that it was not the matrix but actually the protein contained within the matrix that was responsible for ectopic bone formation. The first clinical study was conducted in 1988 by Johnson and associates, ${ }^{7}$ who studied purified human BMP. Intensive competition followed in gene sequencing for the BMP. Two groups, one at Creative BioMolecules and the other at Genetics Institute, simultaneously deduced the gene sequence for various BMPs, which resulted in a patent dispute that was subsequently resolved. The human BMP is now produced by using recombinant techniques. Therefore, the available protein is free from the risk of infection or allergic reaction. The cost of the protein, however, remains high. The final landmark in this saga is the FDA approval in 2002 for OP-1 (BMP-7) for long bone defects (Stryker Corp., Kalamazoo, MI) and BMP-2 in a collagen carrier within a cage for anterior lumbar interbody fusions (Medtronic Sofamor Danek, Memphis, TN).

\section{CLASSIFICATION AND CHEMICAL STRUCTURE OF BONE MORPHOGENETIC PROTEIN}

Bone morphogenic proteins are members of TGF $\beta$ superfamily, a large family of growth factors (Table 4). ${ }^{4,6,31-34}$ The TGF $\beta$ was so named because of its ability to transform cultured fibroblasts. The BMP subfamily com-

TABLE 1

Stages of bone healing and remodeling

\begin{tabular}{ll}
\hline \hline \multicolumn{1}{c}{ Stage } & \multicolumn{1}{c}{ Characterization } \\
\hline $\begin{array}{l}\text { I: induction } \\
\text { II: inflammation }\end{array}$ & $\begin{array}{l}\text { formation of hematoma at fracture site: release of growth factors \& cytokines } \\
\text { recruitment of inflammatory cells, macrophages, \& fibroblasts to the injury site } \\
\text { mitosis of mesenchymal cells and differentiation of chondrocytes; hypertrophy of chondrocytes \& } \\
\text { calcification; deposition of extracellular collagenous matrix; local angiogenesis }\end{array}$ \\
$\begin{array}{ll}\text { IV: woven bone formation } \\
\text { V: lamellar bone formation }\end{array}$ & $\begin{array}{l}\text { differentiation of osteoblasts, mineralization of EM } \\
\text { bone resorption, remodeling, formation of lamellar bone \& hematopoietic marrow }\end{array}$ \\
\hline
\end{tabular}


TABLE 2

Growth factors and cytokines involved in the generation of new bone and remodeling

\author{
BMPs \\ TGF- $\beta$ \\ PDGF \\ insulin-like growth factor SI \& II \\ epidermal growth factor \\ fibroblast growth factor \\ vascular endothelial growth factor \\ Turner necrosis factor
}

prises more than 10 proteins, and newer ones are being discovered. There are several structural homologies between BMPs and TGF $\beta$ growth factors. The amino acid sequence of BMPs is highly conserved, and is considered to be as old as 600 million years. Because of this conservation, human recombinant BMPs are highly effective in lower life forms, including fruit flies. Like all members of the TGF $\beta$ family, BMPs are synthesized as precursor proteins. The precursor protein contains hydrophobic secretive leader sequence as well as substantial propeptides. The mature portion of the protein is located at the carboxy terminal of the precursor molecule. In their carboxy terminal portions, all BMPs contain seven cysteine amino acid residues in positions identical to those present in all members of the TGF $\beta$ superfamily. In addition, BMPs contain N-linked glycosylation sites.

The BMP protein can be broadly classified into three subfamilies. $22,24,25$ Both BMP-2 and BMP-4 have $80 \%$ amino acid sequence homology of molecules. In the second group, consisting of BMP-5, -6 and -7 , the mean is $78 \%$ amino acid sequence homology whereas the third group, composed solely of BMP-3, is significantly different from the other members of the BMP family and generally stands alone. It is of interest that there is a substantial homology between decapentaplegic peptide and BMP-2 and BMP-4 subfamily, implying that these two BMPs are equivalents of decapentaplegic peptide gene products. Human BMP-6 shows $90 \%$ sequence homology across the entire precursor molecule with Vgr-1. There is reason to believe that $\mathrm{Vgr}-1$ is a murine homolog of BMP-6.

The mature segment of the BMPs, which is highly con- served in all organisms, contains seven cysteine amino acid residues. Six of these residues are involved in the formation of intrachain disulphide bonds that forms a rigid "cysteine-knot" molecular structure. The seventh cysteine residue is involved in the formation of dimers via interchain disulphide bond. The dimers may be either homo- or heterodimers. Formation of homo/heterodimers increases the variability of the effector molecule. The reason for this redundancy is not fully understood but probably offers a larger repertoire of molecules with similar functions.

Although BMP is one among the growth factors, it is unique. It is the only morphogen of all known growth factors that has the ability to transform connective tissue cell into osteoprogenitor cells; thus, it is not only a mitogen stimulating the multiplication of connective tissue cells but can be a morphogen, which is able to transform connective tissue cells into osteoprogenitor cells. All other growth factors such as TGF $\beta$, insulin-like growth factor, fibroblast growth factor, PDGF, and vascular endothelial growth factor all induce multiplication of cells but do not transform one cell type into the other.

\section{SIGNALING MECHANISM OF BONE MORPHOGENETIC PROTEINS}

The BMP receptors on the cell surface are made up of Type I and Type II serine/threonine kinase proteins. This receptor protein is unique to the TGF $\beta$ superfamily of growth factors including BMP. The binding of the ligand to the Types I and II serine/threonine kinase transmembrane receptors results in the formation of heterotetramer complex and activation of the signaling cascade. Immediately after the binding, the Type II receptor kinase phosphorylates the Type I receptor. In turn, the Type I receptor phosphorylates the intracytoplasmic signaling molecules Smads 1, 5, and 8. Following the phosphorylation, Smads 1,5 , and 8 bind to Smad 4 and translocate into the cell nucleus. The entry of the Smads 4/phosphorylatedSmad-1, 5, 8 complex into the cell nucleus results in the activation of transcriptional factors for the early BMP response genes. The BMP signaling cascade, however, is far more complex than indicated by this brief description. There seems to be considerable "cross-talk" between signaling molecules of other growth factors with the BMP signaling complex. This reactivity is undergoing elucidation at this time.

TABLE 3

Milestones in the discovery and use of BMPs*

Authors/Company \& Year

Senn, 1889

Levander, 1934 \& 1938

Sharrard \& Collins, 1961

Urist, 1965

Sampath \& Reddi, 1981

Johnson, et al., 1992

Creative BioMolecules \& Genetic Institute, 1990s

Stryker Corp \& Medtronic Sofamor Danek, 2002
Observation/Discovery

$*$ ALIF = anterior lumbar interbody fusion; EDTA = ethylenediaminetetraacetic acid. 
TABLE 4

Bone morphogenetic protein family

\begin{tabular}{ll}
\hline \multicolumn{1}{c}{ BMP } & \multicolumn{1}{c}{ Function } \\
\hline BMP-2 & $\begin{array}{l}\text { osteoinductive, osteoblast differentiation, apotosis } \\
\text { most abundant BMP in bone, inhibits osteogenesis }\end{array}$ \\
BMP-3 (osteogenin) & \\
BMPs & osteoinductive, lung \& eye development \\
BMP-4 & chondrogenesis \\
BMP-5 & $\begin{array}{l}\text { osteoblast differentiation, chondrogenesis } \\
\text { BMP-6 }\end{array}$ \\
BMP-7 (OP-1) & $\begin{array}{l}\text { osteoinductive, development of kidney \& eye } \\
\text { nervous system, hepatic reticuloendothelial sys- } \\
\text { tem, hepatogenesis }\end{array}$ \\
BMP-9 & cardiac development \\
BMP-10 & patterning mesodermal \& neuronal tissues \\
BMP-11 (GDF-8, & induces tendon-iliac tissue formation \\
myostatin) & induces tendon \& ligament-like tissue formation \\
BMP-12 (GDF-7) & chondrogenesis, enhances tendon healing \& bone \\
BMP-13 (GDF-6) & formation \\
BMP-14 (GDF-5) & modifies follicle-stimulating hormone activity \\
BMP-15 &
\end{tabular}

* GDF $=$ growth/differentiation factor.

\section{Dosage of BMP}

It has been estimated that normal bone contains approximately $0.002 \mathrm{mg}$ of BMP per kilogram of pulverized bone. At a fracture site, presumably the BMP is released at a higher concentration because of the secretion by the transformed inflammatory cells into osteoprogenitor cells and upregulation of BMP from the released cytokines at the fracture site. The exact concentration of the BMP at the fracture site as opposed to physiological concentration in the normal bone is unknown.

The concentration required for ideal induced bone bridging in osseous defects depends on several factors. First is the state of the organism in the evolutionary scale. Lower forms such as rodents heal much faster than higher forms such as subhuman primates. Experimental studies involving larger, highly evolved animals require more BMP for the fusion of comparable defects induced in the lower mammals. Additionally, the type of defect should be considered. Simple closed fractures whose ends are apposed by external splinting or internal stabilization do not require any extraneously introduced BMP; is a 99\% fusion rate. Apparently, the enhanced BMP released locally at the site of fracture is sufficient to induce bridging of the broken fragments if they are in apposition. Therefore, the use of BMP is seldom considered when treating straightforward long bone fractures. On the other hand, fractures in which there are critical segmental defects in the long bones do not heal spontaneously. They require adjuvant autograft or BMP for adequate healing.

For spine application in the spine, the interbody fusion is less demanding than intratransverse fusion. The latter is well known to be associated with the highest nonfusion rates. The favorable outcome of interbody fusion is related to the fact that drilling of the cortical endplate exposes the bone marrow at the adjacent opposing surface of the fusion, and there is a contiguous supply of bone marrow cells. The gap is seldom more than $13 \mathrm{~mm}$ between the vertebral bodies in the lumbar region. The vascularity from the osseous surface is significant. The graft is in the compression mode. All of these factors lead to a favorable outcome with regard to the potential for fusion and require smaller doses of BMP.

In contrast, intertransverse fusion is very demanding. Limited surface area is provided for the exposed cancellous bleeding bone. The linear dimension of the bone to be bridged is generally of the order of $2.5 \mathrm{~cm}$. The bone graft is not loaded. All these factors lead to poor outcome, and in this context the largest doses of BMP need to be used $(3-3.5 \mathrm{mg})$.

The authors of dose escalation studies in animal models have indicated that the superphysiological dose approaching 3 to $3.5 \mathrm{mg}$ of BMP is sufficient in virtually all cases to induce new bone and to bridge the osseous defect. Additional doses of BMP do not confer any benefit in terms of fusion rate or the time taken for the fusion to occur. The quality of BMP-induced bone is comparable with and is indistinguishable from the natural bone. The difference in bone healing when applying BMP is that under normal conditions in the abscence of BMP the bone growth occurs from the bone margins of the gap to be filled and progressively creeps from the ends toward the center; whereas with BMP-induced bone, however, is formed concurrently throughout the defect. Thus, BMP transforms inflammatory cells into osteoprogenitor cells that may freely cross the gap in a concurrent fashion and lay down bone simultaneously to close the gap.

Bone induced under the influence of BMP matures faster than natural healing of the bone. In humans, healing is complete by 8 to 10 weeks as opposed to 12 to 16 weeks when autograft is used.

\section{CARRIERS FOR BMP}

Bone morphogenetic protein is a water-soluble relatively low-molecular weight protein that diffuses very easily in the body fluids. When administered in a surgical setting such as in spinal fusion, because the protein will diffuse very rapidly in wound hematomas or can be irrigated away or lost in the suction drainage, it is necessary to contain the BMP. In an experimental setting BMP delivered without a carrier does not endure more than a few hours at the deposited site. It is therefore necessary to contain the BMP in a carrier so that it will have a localized effect at the bone healing site. ${ }^{26}$

The need for a carrier has been recognized since BMP was initially identified. Various carriers have been investigated experimentally and clinically. ${ }^{8}$ The BMP carriers can be broadly classified into inorganic salts, naturally occurring polymeric substances, synthetic polymers, and composites of synthetic and naturally occurring polymers.

An ideal carrier should neither induce an inflammatory response nor immune reaction. Degradation of the carrier should not result in toxic residues. Ideally the carrier should be absorbed concurrent with bone healing, leaving no residue. It should be porous, the porosity being equivalent to cancellous bone. The porosity permits trapping of inflammatory cells and bone growth factors. Debate exists regarding the ideal configuration and the sizes of the porosity needed for bone growth. There are competing claims concerning the intercommunicating nature of the porosity with open ends at the surface and the size of the pores. It 
is generally agreed that the pore size should be at least comparable with the porosity in the cancellous bone. Pore size even larger than that is thought to be beneficial. Whether the pores should be blind pockets or communicating with each other as well as with the environment is somewhat debatable, and there is no definitive answer to this question.

The most commonly used inorganic salts are Ca phosphate and $\mathrm{CaO}_{4} \mathrm{~S}$. The hydroxyapatite, which is a naturally occurring bone mineral and is resorbed rather slowly, ${ }^{21}$ is becoming less popular than the alternate TCP. The TCP granules are extensively used as a bone extender. It is absorbed slightly faster ( 45-60 days) than the rate of formation of the bone. It can be formulated with varying porosities. Although TCP is a commonly used laboratory chemical, induction of the ideal pore size and geometry requires modification in the synthetic process, which can be a patent-protected intellectual property. Although used experimentally, TCP granules have not been extensively used with BMP in a clinical setting. Phosphate cements are not porous and therefore are not considered as carriers for BMP.

Collagen is the most commonly used carrier, and Type I collagen is preferred. This can be obtained from bone or from tendons and ligaments. Bovine collagen is currently used in the clinical setting as a carrier. It has become apparent from practical use, however, that BMP binds tightly to the bone-derived collagen and not to the tendonderived collagen. Therefore, the use of BMP-2, which has been approved by FDA for human use, is restricted. Because BMP can be easily squeezed out of the collagen by axial loads and under pressure, it has to be contained in a cage for interbody fusion. Due to fear of the BMP escaping into the epidural space and causing unwanted bone in the epidural location, it has not yet been approved for intertransverse fusion. Osteogenic protein-1 or BMP-7 uses bone-derived collagen, which binds strongly to the BMP presumably through hydrogen bonding. Because of this tighter bonding, OP-1 does not require containment in a cage. Currently OP-1 is used only for long bone defects. It has not been approved for applications in the spine. Demineralized bone matrix as a carrier has not gained popularity because of the risk of immunogenicity and the risk of disease transmission. Other natural polymers that have been considered as a carrier are hyalurone, fibrin, chitoson, alginate, and other animal- or plant-derived polysaccharides. None of these has gained acceptance for human use at the time of this writing.

Synthetic polymers carry the advantage of abundant unlimited supply, low or no antigenicity, predictable absorption, and no risk of disease transmission. Although polyglycolic acid and polylactic acid derivatives have been explored, their degradation products can produce giant cell reaction; the binding affinity of BMP to the synthetic polymers is not as good as that for collagen. Therefore, at present synthetic polymers are not used extensively in humans but are likely to be used when advances in polymer technology occur.

In summary, the most commonly used carrier for human use is Type I collagen. Bone-derived collagen appears to have an advantage over collagen-derived from tendons and ligaments because of tighter binding.

\section{GENE THERAPY}

Delivery of the $B M P$ gene as a transgene for the target cells remains a potential strategy. ${ }^{5}$ The genetic material can be delivered either via a nonreplicating viral vector or nonviral vector. A consistent problem associated with gene therapy has been the low transduction rate. In addition, sustained expression has been difficult to achieve, although with BMP and other growth factors sustained lifetime expression is not needed. Rather, it is needed only throughout the bone healing process. Nonetheless, considerable work is required before gene therapy becomes a reality in a clinical setting.

\section{LONG TERM CONCERNS WITH BMP}

There are several long-term concerns about the use of recombinantly manufactured BMPs in humans. ${ }^{12}$ The effects of high doses of BMP on a developing embryo are unknown. Therefore, at this time its use during pregnancy is not advised. Although it is a human protein, there is a risk of developing an immune reaction to the protein. This risk increases if BMP is administered more than once such as in cases of repeated fusion. The magnitude of this risk remains unknown.

Development of an osteogenic sarcoma is possible, although experimental dose escalation studies in animal models have not induced neoplasm. It is of some concern that spontaneously evolving osteosarcomas contain high levels of BMPs.

Uncontrollable bone growth in the vicinity of the neural structures, especially nerve roots and cauda equina, is a potential problem. This can only be solved using efficient carriers that bind the protein tightly, preventing BMP release during of protein axial loading.

\section{OTHER USES OF BMP}

Bone morphogenetic protein affects organ systems other than bone. ${ }^{1}$ It is believed to be a brain protective agent. In fact, in an ischemic rat model, BMP has been shown to offer protection by reducing the size of the infarct. It is an intriguing possibility that in the future BMP may be useful as a protective agent in severe head trauma and stroke.

In patients with chronic renal disease levels of BMP are lower because kidneys are their primary source in the human adult. It is possible that systemic administration of BMP may restore some of the renal functions in patients with chronic renal failure. Additionally, spine surgeons are all too familiar with the renal osteodystrophy syndrome that occurs in patients undergoing long-term dialysis in cases of endstage kidney disease. Fusion rates in these individuals are dismal. Application of BMP locally may be beneficial.

There is considerable ongoing research to determine whether a combination of growth factors (other than BMP) and demineralized bone matrix along with bone marrow aspirate will prove an appropriate substitute for BMP. Recent experimental results are encouraging, but large clinical trials are required to determine if combination therapy will be as efficacious as recombinantly derived human BMP for inducing osseous fusion. 


\section{CONCLUSIONS}

The cellular and molecular events governing bone formation in the embryo, healing of a fractured bone, and induced bone fusion follow virtually identical patterns. The discovery, purification, and synthesis of BMPs involving recombinant techniques constitutes a major milestone in the understanding of bone physiology. The mechanism of action, clinical application, dosage, and optimum carriers for the protein have been discussed. The roles played by other growth factors have also been discussed.

\section{References}

1. Azari K, Doll BA, Sfeir C, et al: Therapeutic potential of bone morphogenetic proteins. Expert Opin Investig Drugs 10: 1677-1686, 2001

2. Barnes GL, Kostenuik PJ, Gerstenfeld LC, et al: Growth factor regulation of fracture repair. J Bone Miner Res 24:1805-1815, 1999

3. Einhorn TA: The cell and molecular biology of fracture healing. Clin Orthop 355 (Suppl):7-21, 1998

4. Elima K: Osteoinductive proteins. Ann Med 25:395-402, 1993

5. Helm GA, Alden TD, Sheehan JP, et al: Bone morphogenetic proteins and bone morphogenetic protein gene therapy in neurological surgery: a review. Neurosurgery 46:1213-1222, 2000

6. Hoffmann A, Weich HA, Gross G, et al: Perspectives in the biological function, the technical and therapeutic application of bone morphogenetic proteins. Appl Microbiol Biotechnol 57: 294-308, 2001

7. Johnson EE, Urist MR, Finerman GA: Resistant nonunions and partial or complete segmental defects of long bones. Treatment with implants of a composite of human bone morphogenetic protein (BMP) and autolyzed, antigen-extracted, allogeneic (AAA) bone. Clin Orthop 277:229-237, 1992

8. Kirker-Head CA: Potential applications and delivery strategies for bone morphogenetic proteins. Adv Drug Deliv Rev 43: 65-92, 2000

9. Levander G: On the formation of new bone in bone transplantation. Acta Chir Scand 74:425-426, 1934

10. Levander G: A study of bone regeneration. Surg Gynecol Obstet 67:705-714, 1938

11. McKibbin B: The biology of fracture healing in long bones. J Bone Joint Surg Br 60:150-162, 1978

12. Poynton AR, Lane JM: Safety profile for the clinical use of bone morphogenetic proteins in the spine. Spine 27 (Suppl 1): 40-48, 2002

13. Probst A, Spiegel HU: Cellular mechanisms of bone repair. J Invest Surg 10:77-86, 1997

14. Ray RD, Holloway JA: Bone implants. Preliminary report of an experimental study. J Bone Joint Surg Am 39:1119-1128, 1957

15. Reddi AH: Bone and cartilage differentiation. Curr Opinion Genet Dev 4:737-744, 1994

16. Reddi AH: Cell biology and biochemistry of endochondral bone development. Coll Relat Res 1:209-226, 1981
17. Reddi AH: Role of morphogenetic proteins in skeletal tissue engineering and regeneration. Nat Biotechnol 16:247-255, 1998

18. Reddi AH, Ma SS, Cunningham NS: Induction and maintenance of new bone formation by growth and differentiation factors. Ann Chir Gynaecol 77:189-192, 1988

19. Reddi AH, Wientroub S, Muthukumaran N: Biologic principles of bone induction. Orthop Clin N Am 18:207-212, 1987

20. Ripamonti U, Duneas N: Tissue morphogenesis and regeneration by bone morphogenetic proteins. Plast Reconstr Surg 101:227-239, 1998

21. Ripamonti U, Ma S, Reddi AH: The critical role of geometry of porus hydroxyapatite delivery system in induction of bone by osteogenin, a bone morphogenetic protein. Matrix 12: 202-212, 1992

22. Sakou T: Bone morphogenetic proteins: from basic studies to clinical approaches. Bone 22:591-603, 1998

23. Sampath TK, Reddi AH: Dissociative extraction and reconstitution of bone matrix components involved in local bone differentiation. Proc Natl Acad Sci USA. 78:7599-7603, 1981

24. Schaub RG, Wozney J: Novel agents that promote bone regeneration. Curr Opin Biotechnol 2:868-871, 1991

25. Schmitt JM, Hwang K, Winn SR, et al: Bone morphogenetic proteins: an update on basic biology and clinical relevance. J Orthop Res 17:269-278, 1999

26. Seeherman H, Wozney J, Li R: Bone morphogenetic protein delivery systems. Spine 27 (Suppl 1):16-23, 2002

27. Senn N: On the healing of aseptic bone cavities by implantation of antiseptic decalcified bone. Am J Med Sci 98:219-243, 1889

28. Sharrard WJ, Collins DH: The fate of human decalcified bone grafts. Proc Roy Soc Med 54:1101-1102, 1961

29. Solheim E: Growth factors in bone. Int Orthop 22:410-416, 1998

30. Urist MR: Bone: formation by autoinduction. Science 150: 893-899, 1965

31. Vukicevic S, Stavljenic A, Pecina M: Discovery and clinical applications of bone morphogenetic proteins. Eur J Clin Chem Clin Biochem 33:661-671, 1995

32. Wozney JM: The bone morphogenetic protein family and osteogenesis. Mol Reprod Dev 32:160-167, 1992

33. Wozney JM: Bone morphogenetic proteins. Prog Growth Factor Res 1:267-280, 1989

34. Wozney JM: The bone morphogenetic protein family: multifunctional cellular regulators in the embryo and adult. Eur J Oral Sci 106 (Suppl 1):160-166, 1998

35. Wozney JM, Rosen V, Byrne M, et al: Growth factors influencing bone development. J Cell Sci Suppl 13:149-156, 1990

36. Yoo JU, Johnstone B: The role of osteochondral progenitor cells in fracture repair. Clin Orthop 355 (Suppl):73-81, 1998

Manuscript received November 29, 2002.

Accepted in final form December 2, 2002.

Address reprint requests to: Setti S. Rengachary, M.D., Department of Neurosurgery, 4201 Saint Antoine, Suite 6E, Detroit, Michigan 48201. email address: srengachary@ neurosurgery.wayne.edu. 